\title{
Management of Percutaneous Core Biopsy Tract Bleeding
}

\section{Pranjali V. Gadgil and V. Suzanne Klimberg}

Division of Breast Surgical Oncology, Department of Surgery, University of Arkansas for Medical Sciences, Little Rock, AR 72205-7199 